\title{
Satellite Image Resolution Enhancement Using Non-Decimated Wavelet Transform and Gaussian Mixture Model
}

\author{
T. V. Hyma Lakshmi \\ Assistant Professor, S. R. K. R. Engineering College, Bhimavaram, India. \\ T. Madhu \\ Principal, SIET, Narasapur, India, \\ E V Krishna Rao \\ Professor, Dept of ECE, L B R College of Engg., Mylavaram-521230, AndhraPradesh, INDIA,
}

\section{K CH Srikavya}

Professor, Dept of ECE, K L University, India

\begin{abstract}
High resolution satellite images are of significant importance in many fields of research and its enhancement is an active research area. Among various techniques used for image resolution enhancement Wavelet based techniques have been found to be very efficient. Discrete Wavelet Transform (DWT) is mostly used in image decomposition stage but suffers from shift variance. In this work Non-Decimated Wavelet Transform (NDWT) which is shift invariant is investigated. Instead of Bicubic interpolation Gaussian Mixture Model (GMM) which is a parametric probability density function represented as a weighted sum of Gaussian component densities is used instead of weighted sum of neighborhood pixels used by bicubic and bilinear interpolations. The proposed technique showed improved Peak Signal to Noise Ratio (PSNR) and Quality Index.
\end{abstract}

Keywords: Bicubic Interpolation; Discrete Wavelet Transform (DWT); Gaussian Mixture Model (GMM); NonDecimated Wavelet Transform (NDWT); Peak Signal-toNoise Ratio (PSNR); Quality Index (QI);

\section{Introduction}

Satellite images are used in many applications such as geoscience studies, weather forecasting, astronomy and geographical information systems [1]. However, high resolution satellite images are essential for better results and thus image resolution enhancement plays a crucial role. Popular techniques used in literature include nearest neighbour interpolation, bilinear interpolation and bicubic interpolation [2]. Bicubic interpolation is widely compared to the other two techniques and it produces noticeably sharper images [3]. When applying interpolation methods, the high frequency components may be eliminated because of the smoothing effects created during interpolation. It is imperative that pixel values around the edges be preserved to improve the resolution of the image. Wavelets play a vital role in image resolution enhancement techniques to preserve the edges. Discrete Wavelet Transform (DWT) is used in image decomposition stage and bicubic interpolation is used in interpolation stage in many of the wavelet based image resolution enhancement methods [4][5]. DWT decomposes the image into four sub band images defined as Low-Low (LL), Low-High (LH), High-Low (HL), and High-High (HH). The frequency components of these sub bands cover the full frequency spectrum of the original image. Theoretically, a filter bank should be operated on the image in order to generate different sub band frequency images. Edges identified in lower frequency sub bands are used to prepare the model for estimating edges in higher frequency sub-bands, and only the coefficients with significant values are estimated as the evolution of the wavelet coefficients. Filter bank of DWT is shown in figure1 [6].

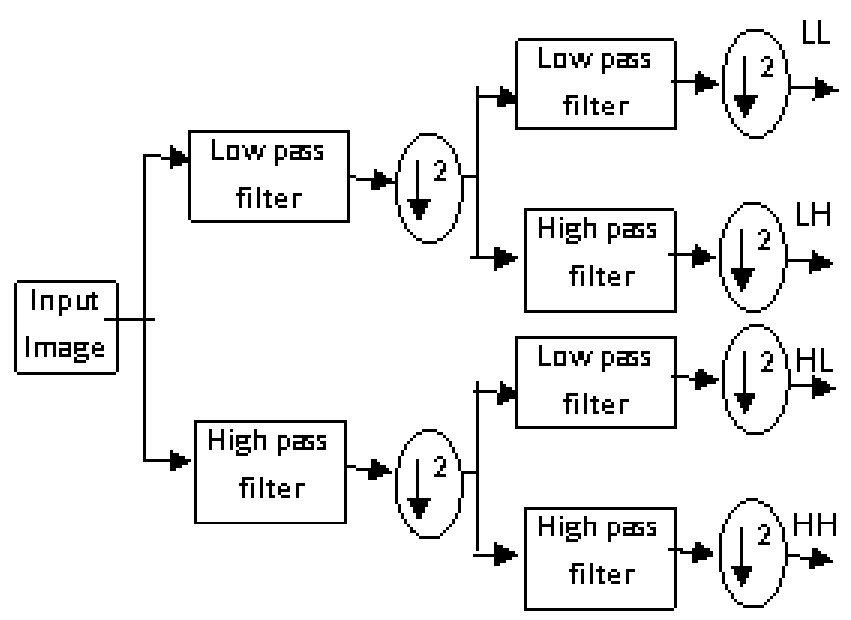

Figure 1. Filter Bank of DWT 
Hasan et al [4] proposed a resolution enhancement technique based on the image decomposition using DWT and coefficients are interpolated using bicubic interpolation. When tested on satellite benchmark images the quantitative and visual results showed that their proposed technique was superior to the conventional image resolution enhancement techniques. Battulla et al., [7], Mohan, et al., [8] and Karunakar et al., [9] also proposed DWT decomposition and interpolation of high frequency components. Salehi and Nasab presented image resolution improvement method based on the complex wavelet transform and feed forward Neural Networks (NN) [10]. The wavelet sub bands of high resolution images are constructed by using the $\mathrm{NN}$ using the low resolution sub bands. Dual complex tree properties such as approximate shift variance, directional selectivity and substantial reduced aliasing were used to get detailed representation of the local structures in the interpolated images.

Zhang [11] proposed edge-guided nonlinear interpolation method by using a directional filtering and data fusion. To interpolate a pixel, two observation sets are created in two orthogonal directions, and each set produces an estimate of the pixel value. These estimated sets were modeled as different noisy measurements in missing pixels and fused by the Linear Minimum Mean Square-Error Estimation (LMMSE) technique using the statistics. Experiments show that the proposed interpolation method preserved sharpness of edges and reduced the ringing artifacts. Huayong et al., [12] proposed a novel method for single image super-resolution. From the input low-resolution images, a pyramid pair was constructed by using the ground truth pyramid and the interpolated pyramid. The relationship between pixel value in ground truth pyramid and its corresponding 8-neighborhood vector in interpolated pyramid was found by using GMM. All the pixel values of the high-resolution image were estimated by using the corresponding 8-neighborhood vector through the trained GMM. Taquet et al., [13] proposed an interpolation scheme based on Gaussian mixture simplification and demonstrated the advantages over a heuristic approach by means of the spatial normalization and tractography results by using Diffusion Tensor Imaging. The results were compared with the conventional bi linear and bi cubic interpolation methods and it was demonstrated that their proposed method was better as it accurately reconstructed the edges and textures at low cost. Hou et al [14] proposed two synthetic aperture radar complex image compression schemes based on Directional Lifting Wavelet Transform Image Quality (DLWT_IQ) and DLWT_ Fast Fourier Transform (DLWT_FFT). The real parts and imaginary parts of the images are encoded by DLWT_IQ and the real images are converted by FFT is encoded by DLWT_FFT.

DWT suffers from shift variance and bicubic interpolation gives rise to blurred edges. To overcome this, a new image resolution enhancement technique Non-Decimated Wavelet Transform (NDWT) image decomposition and Gaussian Mixture Model (GMM) interpolation is proposed in this paper. Each sub band within the NDWT decomposition has the same number of coefficients as the number of samples in the original signal, thus leading to complete representation and completely shift invariant. GMM is a flexible, semi- parametric model, yet simple model which makes efficient estimations.

The flow chart for Satellite Image Resolution Enhancement using DWT and interpolation using Bicubic and Gaussian Mixture Model (GMM) is shown in figure2. Here DWT is used to decompose an input image into Low Low (LL), Low High (LH), High Low (HL) and High High (HH) sub bands. These sub bands are interpolated using Bicubic interpolation technique, followed by combining all these images to generate a new high-resolution image by using inverse DWT. Results of this technique are compared with proposed technique.

When applying interpolation methods, the high frequency components may be lost as the coefficients of DWT are inherently interposable. But Down sampling in each of the DWT sub bands will cause loss of information in the respective sub bands. DWT is a non redundant transform and artifacts such as ringing are introduced when transform coefficients are modified and this can be eliminated by using NDWT.

This paper is organized as follows: Section2 gives an overview on the bicubic interpolation, Section 3 introduces the proposed NDWT and GMM interpolation technique and Section 4 discusses the visual and quantitative results of the proposed techniques. The results of the proposed method are compared with other conventional techniques. Both quantitative and visual results show the superiority of the proposed technique. Conclusions are given in the final section.

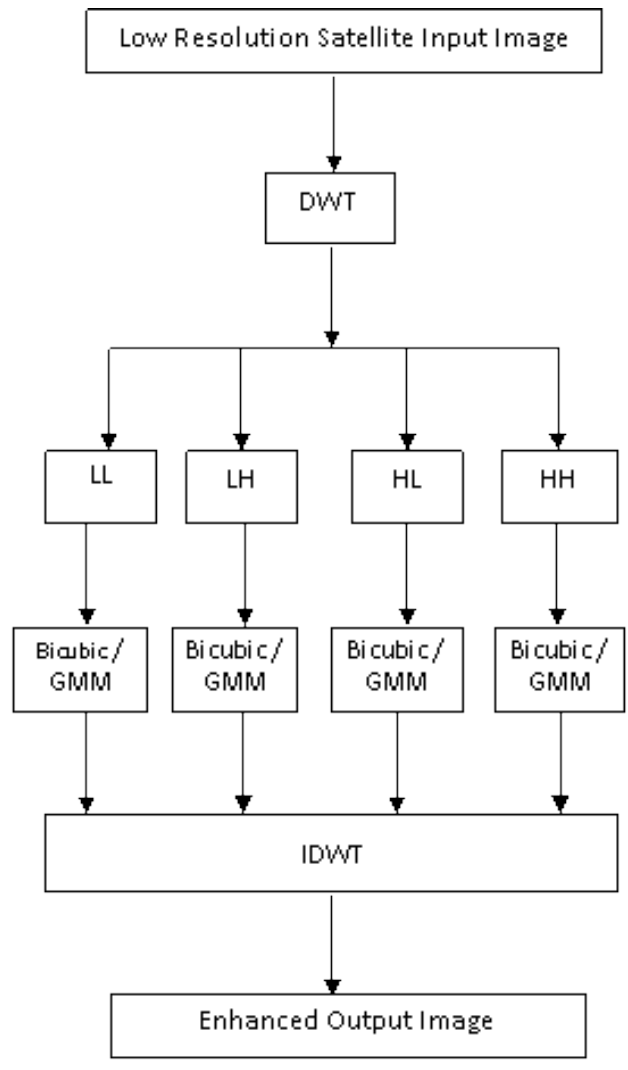

Figure 2. Block diagram of Image decomposition using DWT and Bi-cubic / GMM interpolation Satellite Image Resolution Enhancement Technique. 


\section{Bicubic Interpolation}

Interpolation is a commonly used method for resolution enhancement. In linear interpolation method, mean value of neighbouring pixels is used to interpolate at each pixel, but it may create blurred edges and smoothed details. In bilinear interpolation, new pixel value is computed by weighted average of four surrounding pixels. This will be useful for image compression instead of image resolution enhancement to reduce the redundancy. Many non-linear interpolation (bicubic) methods are more powerful than linear methods (bilinear) [7]. However, if the raw image has more lowerfrequency information, it is better to use the bilinear interpolation rather than bicubic interpolation. In bicubic interpolation, interpolated point is filled with sixteen closest pixel's weighted average [7]. The bicubic convolution interpolation kernel is:

Where $a$ is generally taken as- 0.5 to- 0.75

\section{Proposed Technique}

In this section NDWT and GMM is discussed followed by the proposed technique.

\section{1 Non Decimated Wavelet Transform}

The Discrete Wavelet Transform is not a time invariant transform. The way to restore the translation invariance is to average some slightly different DWT, called Non-decimated or un-decimated DWT (NDWT or UDWT). It does so by suppressing the down-sampling step of the decimated algorithm and instead of up-sampling the coefficients are interpolated by inserting zeros between the filter coefficients. As with the decimated algorithm, the filters are applied first to the rows and then to the columns. In this case, however, although the four images produced (one approximation and three detail images) are at half the resolution of the original and they are the same size as the original image. The approximation images from the non-decimated algorithm are therefore represented as levels in a parallelepiped, with the spatial resolution becoming coarser at each higher level and the size remaining the same. The 2D NDWT is based on the idea of no decimation. It applies the DWT and omits both down-sampling in the forward and up-sampling in the inverse transform. More precisely, it applies the transform at each point of the image and saves the detail coefficients and uses the low frequency information at each level [15].

The Non Decimated Wavelet Transform ' $W$ ' using the filter bank $h, g$ representing high pass and low pass respectively of a 1-D signal $\mathrm{C}_{0}$ leads to a set $W=\left\{w_{i}, \ldots \ldots \ldots, w_{j}, c_{j}\right\}$ where $\mathrm{w}_{\mathrm{j}}$ are the wavelet coefficients at scale $\mathrm{jand} \mathrm{c}_{\mathrm{j}}$ are the coefficients at the coarsest resolution. The passage from one resolution to the next one is obtained using the "à trous" algorithm.

$$
\begin{aligned}
& c_{j+1}[l]=\left(h^{\prime}(j) c_{j}\right)[l]=\sum_{k} h[k]_{j}\left[l+2^{k} k\right] \\
& w_{j+1}[l]=\left(\bar{g}^{(j)} * c_{j}\right)[l]=\sum_{k} g[k] c_{j}\left[l+2^{j} k\right]
\end{aligned}
$$

Here $h^{(j)}[l]=h[l]$ if $l / 2^{j}$ is an integer and zero. For example

$$
h^{(1)}=(\ldots, h[-2], 0, h[-1], 0, h[0], 0, h[1], 0, h[2], 0, \ldots)
$$

The reconstruction is done by,

$$
c_{j}[l]=\frac{1}{2}\left[\left(\tilde{h}^{(j)} * c_{j+1}[l]\right)+\left(\tilde{g}^{(j)} * w_{j+1}[l]\right)\right]
$$

The filter bank (h, g) and $(\widetilde{h}, \tilde{g})$ has to check the reconstruction condition given by,

$$
H\left(z^{-1}\right) \tilde{H}(z)+G\left(z^{-1}\right) \tilde{G}(z)=1
$$

This provides us with a higher degree of freedom when designing the synthesis prototype filter bank. The à trous algorithm can be extended to 2D by

$$
\begin{aligned}
& c_{f+1}[k, l]=\left(\bar{h}(f)-(s) * c_{f}\right)[k, l] \\
& w_{f+1}^{1}[k, l]=\left(\bar{g}(f)-(f) * c_{f}\right)[k, l] \\
& w_{f+1}^{3}[k, l]=\left(\bar{h}(f)-(f) * c_{f}\right)[k, l] \\
& w_{f+1}^{1}[k, l]=\left(g^{(f)}-(f) * c_{f}\right)[k, l]
\end{aligned}
$$

Where hg ${ }^{*} \mathrm{c}$ is the convolution of $\mathrm{c}$ by the separable filter hg (i. e., convolution first along the columns by $\mathrm{h}$ and then convolution along the rows by g). At each scale, we have three wavelet images $w^{1}, w^{2}, w^{3}$ and each has the same size as the original image [16]. For a real discrete-time filter whose impulse response is $h[n], \bar{h}[n]=h[-n], n \in z$ is its timereversed version. For the octave band non sub sampled wavelet representation, analysis and synthesis filters are denoted by $(\mathrm{h}, \mathrm{g})$ and $(\tilde{\mathrm{h}}$ and $\tilde{\mathrm{g}})$ respectively will be used for the Fourier transform of square-integral signals.

\section{2 Gaussian Mixture Model}

Edge structures preservation is a challenging task during the interpolation of images while reconstructing a high-resolution image using low-resolution counterpart. In this proposed technique, input image is decomposed with NDWT and coefficients are interpolated with the highest probability of covariance matrix $\Sigma i$ and mean $\mu \mathrm{i}$ rather than weighted average of sixteen neighbour pixels used in bicubic interpolation. So it gives sharper edges and better PSNR and QI than bicubic interpolation.

A GMM is a parametric probability density function represented as a weighted sum of Gaussian component densities. GMMs are commonly used as a parametric model of the probability distribution of continuous measurements. The complete Gaussian mixture model is parameterized by the mean vectors, covariance matrices and mixture weights from all component densities. The covariance matrices can be full rank or constrained to be diagonal. Additionally, parameters can be shared, or tied, among the Gaussian components, such as having a common covariance matrix for all components. The choice of model configuration including number of components, full or diagonal covariance matrices, and parameter tying are determined by the amount of data available for estimating the GMM parameters and how the GMM is used. A Gaussian mixture model is a weighted sum of $\mathrm{M}$ component Gaussian densities [17] is represented by equation (10). 


$$
p(x \mid \lambda)=\sum_{i=1}^{M} w_{i} g\left(x \mid \mu_{i}, \sum_{i}\right)
$$

Where $x$ is a D-dimensional continuous-valued data is vector and depends on the number of dimensions in the data. $\lambda$ represents the parameterized model of the Gaussian mixture and made up of mean vectors, covariance matrices and mixture weights $\left\{\mu_{i}, w_{i}, \sum_{i}\right\}$

In the case of gray scale image $\mathrm{D}=2 . \mathrm{w}_{\mathrm{i}}, \mathrm{i}=1, \ldots, \mathrm{M}$, mixture weights.

$\mathrm{g}\left(\mathrm{x} \mid \mu \mathrm{i}, \_\mathrm{i}\right), \mathrm{i}=1, \ldots, \mathrm{M}$, Gaussian densities component. Density component is a D-variate Gaussian function consisting of D dimensions given as,

$g\left(x \mid \mu_{i}, \sum_{i}\right)=\frac{1}{(2 \pi)^{D / 2}\left|\sum_{i}\right|^{1 / 2}} \exp \left\{-\frac{1}{2}\left(x-\mu_{i}\right)^{\prime} \sum_{i}^{-1}\left(x-\mu_{i}\right)\right\}$

Where $\mu_{\mathrm{i}}$-mean vector and it is given as $\mu_{\mathrm{i}=} \frac{\sum_{i=1}^{n} x_{i}}{n}$

and $\Sigma_{i}$ is a covariance matrix and it is given as

$\Sigma_{\mathrm{i}}=\frac{\sum_{\mathrm{i}=1}^{\mathrm{n}}\left(\mathrm{x}_{\mathrm{i}}-\overline{\mathrm{x}}\right)}{\mathrm{n}-1}$

In probability theory, covariance is a measure of how much two random variables change together. The mixture weights satisfy the constraint $\sum_{i=1}^{M} w_{i}=1$. Thus, the Gaussian mixture model is represented by the mean vectors, covariance matrices and mixture weights of all component densities. The problem is formulated to the prediction of wavelet coefficients of an image and use the inverse wavelet transform resulting in increased resolution. Training of the mixture model is achieved using Expectation Maximization (EM) algorithm. For a given wavelet coefficient ' $c$ ' that is governed by a group of parameters $\theta$ the density function is given by $\mathrm{p}(\mathrm{x} \mid \theta)$. Let $\mathrm{N}$ be the size of the coefficients and assuming each coefficient is independent and identically distributed with distribution $\mathrm{p}$, the resulting likelihood can be given by

$\mathrm{p}(\mathrm{C} \mid \theta)={ }_{\mathrm{i}=1}^{\mathrm{N}} \mathrm{p}\left(\mathrm{c}_{\mathrm{i}} \mid \theta\right)=\ell(\theta \mid \mathrm{C})$

The function $\ell(\theta \mid C)$ is called the likelihood of the given input wavelet coefficients.

Using EM algorithm it is possible to find additional values. Assuming the coefficients $\mathrm{C}$ is generated by some distribution, it can be stated $\mathrm{C}$ is the incomplete and assuming a complete data $E=(C, D)$ exists such that the joint density function is given by

$\mathrm{p}(\mathrm{E} \mid \theta)=\mathrm{p}(\boldsymbol{c}, \boldsymbol{d} \mid \theta)=\boldsymbol{p}(\boldsymbol{d} \mid \boldsymbol{c}, \theta) \boldsymbol{p}(\boldsymbol{c} \mid \theta)$

The EM algorithm finds the expected value and is given by $\mathrm{O}\left(\theta, \theta^{(\mathrm{i}-1) c}\right)=\boldsymbol{E} \log p(C, D \mid \theta) \mid C, \theta^{(\mathrm{i}-1)}$

Where $\theta^{(\mathrm{i}-1)}$ are the current parameter estimates used to evaluate expectation. The proposed technique is shown as in figure 3

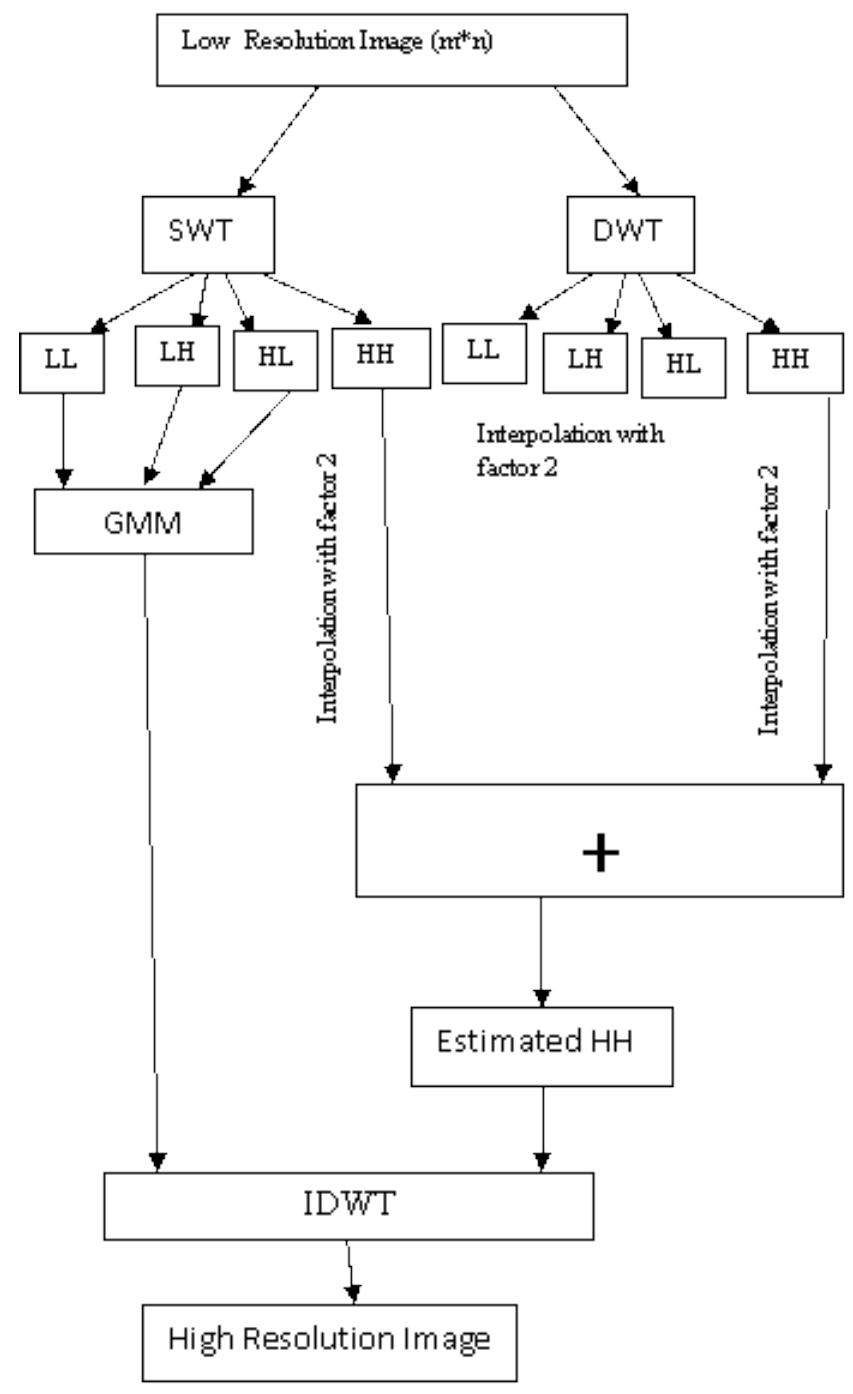

Figure 3: Proposed architecture

\section{Experimental Results}

In order to show the improvement in the resolution of satellite images of the proposed method over the conventional and state-of-art image resolution enhancement techniques, two satellite images with different features are used for comparison. Fig. 4 and Figure. 5 show that high resolution images using the proposed techniques in (f) and (h) are much sharper than the original low-resolution images in (a), bilinear interpolation in (b), bicubic interpolation in (c) wavelet zero padding in (d), DWT based image decomposition and bicubic interpolated images in (e). The proposed technique is evaluated in terms of Peak Signal to Noise Ratio (PSNR), and Quality Index (QI) and compared with other techniques. It is clear that the proposed DWT-GMM and NDWT-GMM techniques outperform than bilinear interpolation, bicubic interpolation, wavelet zero padding and DWT based image decomposition and bicubic interpolated techniques. 


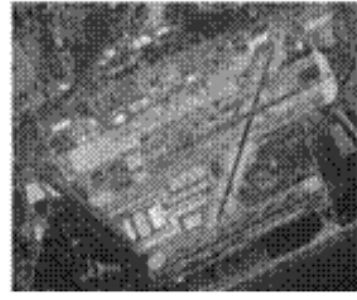

(a)

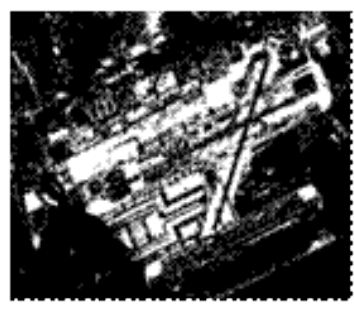

(c)

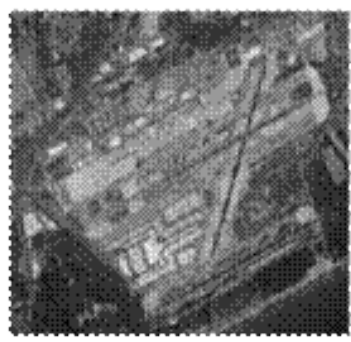

(e)

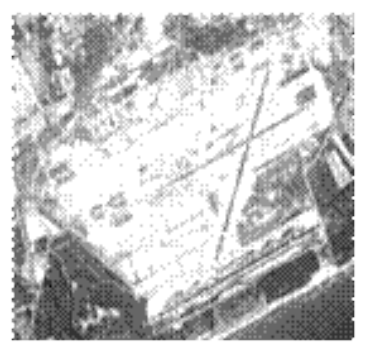

(g)

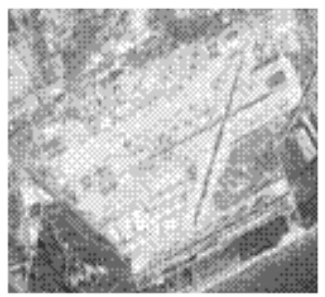

(b)

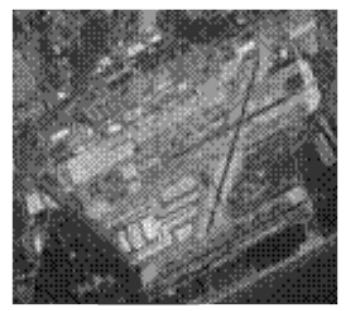

(d)

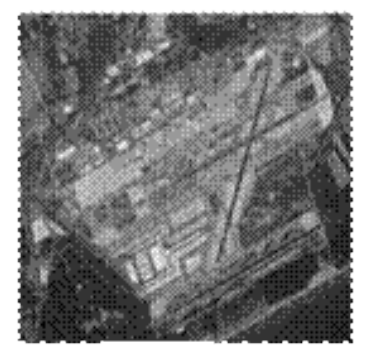

(i)

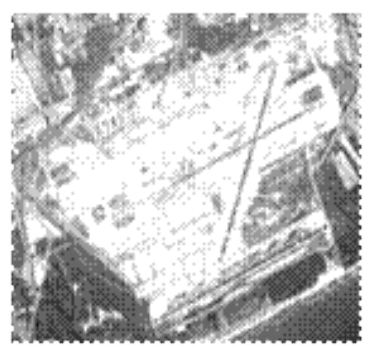

(h)
Figure 4. Satellite Image of US-Topo (a) Low Resolution Input Image, Resolution Enhanced Images using (b) Bilinear Interpolation, (c) Bicubic Interpolation, (d) Wavelet Zero Padding, (e) DWT based Image decomposition and bicubic interpolated image. (f) DWT based Image decomposition and GMM interpolated image, (g) NDWT based decomposition and bicubic interpolatedimage. (h) Proposed Technique (NDWT based decomposition and GMM interpolated) image.
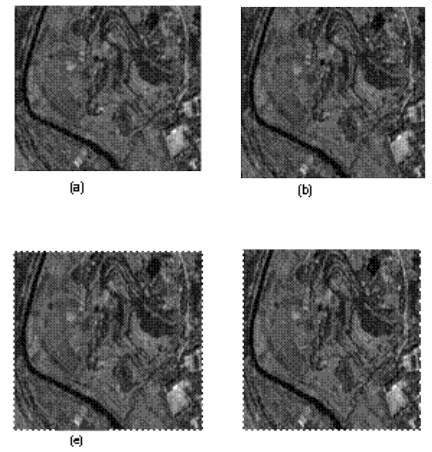
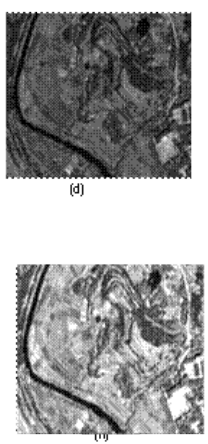

Figure. 5. Satellite image of Washington-DC (a) Low resolution input image, Resolution enhanced images using (b) Bilinear Interpolation, (c) Bicubic Interpolation, (d) Wavelet Zero Padding, (e) DWT based decomposition and bi-cubic interpolated image. (f) DWT based decomposition and GMM interpolated image, (g) NDWT based decomposition and bi-cubic interpolated image. (h)Proposed Technique (NDWT based decomposition and GMM interpolated image).

The PSNR is calculated as follows:

PSNR is the ratio of the maximum possible power of a signal and the power of noise and is expressed in logarithmic decibel scale 10 .

$P S N R=10 \log _{10} \frac{255^{2}}{M S E}$
$M S E=\frac{\sum_{i=1}^{X} \sum_{j=1}^{Y}\left(a_{i, j}-b_{i, j}\right)^{2}}{\mathrm{xy}}$

MSE is a Mean Square Error, where the termsa $a_{i, j}$ and $b_{i, j}$ represent the pixel values from actual and the interpolated images respectively and. the values $\mathrm{X}$ and $\mathrm{Y}$ define the height and width of an image respectively.

And the Quality Index is calculated as below:

Let $x=\left\{x_{i} \mid i=1,2, \ldots, N\right\}$ and $y=\left\{y_{i} \mid i=1,2, \ldots, N\right\}$ be the original and the test image signals respectively. The proposed quality index is defined as

$$
Q=\frac{4 \sigma_{x y} \bar{x} \bar{y}}{\left(\sigma_{x}^{2}+\sigma_{y}^{2}\right)\left[(\bar{x})^{2}+(\bar{y})^{2}\right]}
$$

Where $\bar{x}=\frac{1}{N} \sum_{i=1}^{N} x_{i}, \bar{y}=\frac{1}{N} \sum_{i=1}^{N} y_{i}$

$$
\begin{aligned}
& \sigma_{x}^{2}=\frac{1}{N-1} \sum_{i=1}^{N}\left(x_{i}-\bar{x}\right)^{2} \quad \sigma_{y}^{2}=\frac{1}{N-1} \sum_{i=1}^{N}\left(y_{i}-\bar{y}\right)^{2}, \\
& \sigma_{x y}=\frac{1}{N-1} \sum_{i=1}^{N}\left(x_{i}-\bar{x}\right)\left(y_{i}-\bar{y}\right) .
\end{aligned}
$$

Figure 6 and 7 show the PSNR for the Satellite Image of USTOPO and Satellite image of Washington-DC respectively. The PSNR of the proposed technique is $6.2544 \mathrm{~dB}$ and 4.66 higher than the PSNR of DWT and bicubic interpolation technique respectively.

The Q index of proposed technique is 1.9193 and $1.9853 \mathrm{~dB}$ higher than the Q index obtained by using DWT and bicubic interpolation techniques for image 1 and image 2 respectively. 
Results indicate the superiority of the proposed technique over the conventional and image resolution enhancement techniques.

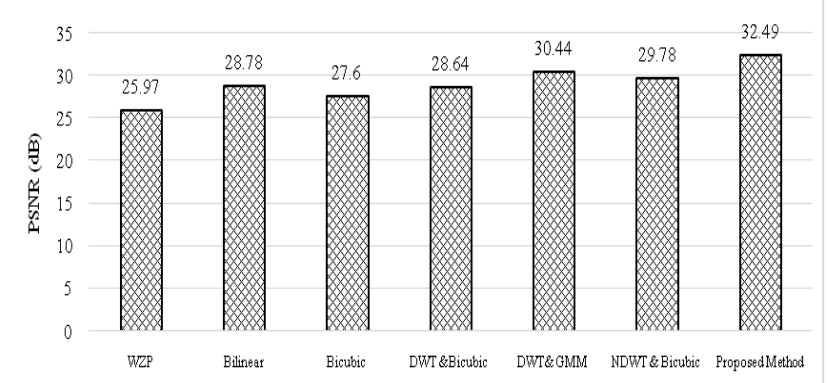

Figure 6 PSNR (Decibels) results for resolution enhancement for the proposed technique compared with conventional and some state-of-art techniques.

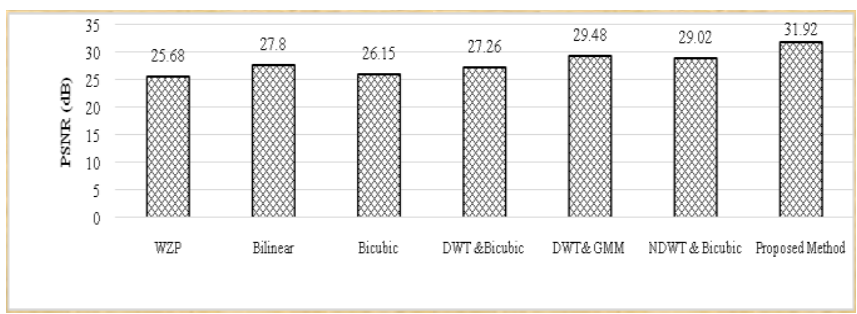

Figure 7 PSNR for Satellite Image of Washington-DC.

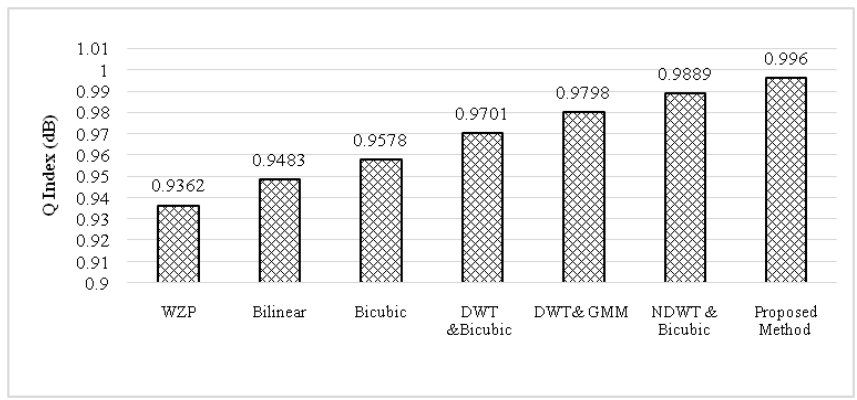

Figure $8 \mathrm{Q}$ index for Satellite Image of US-TOPO.

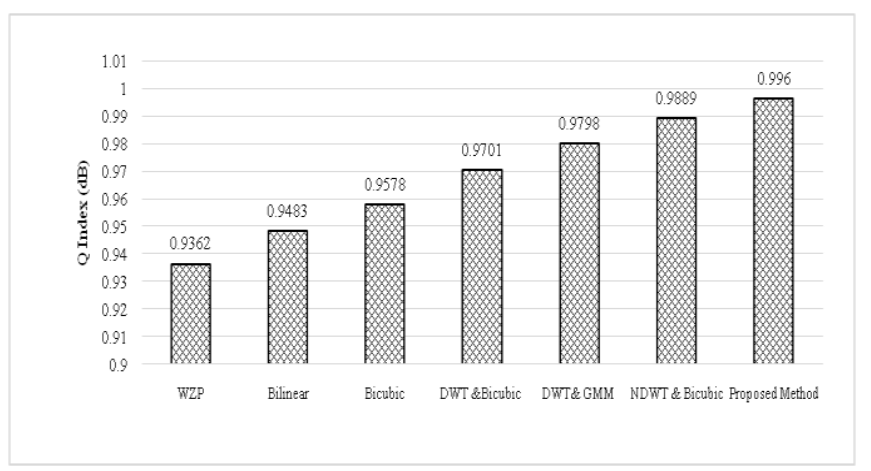

Figure 9 Q index for Satellite Image of Washington-DC.
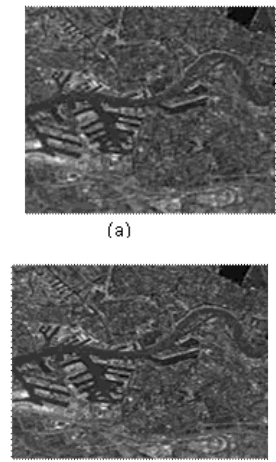

(d)

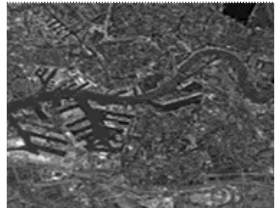

(b)

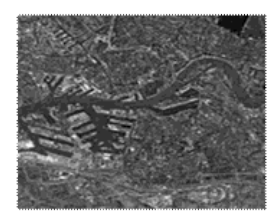

(e)
Figure 10 Satellite Images (a) original low resolution image, (b) DWT based decomposition and bi-cubic interpolated, (c) DWT based decomposition and GMM interpolated image, (d) NDWT based decomposition and bi-cubic interpolated Image and (e) NDWT based decomposition and GMM interpolated image.

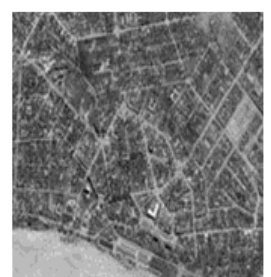

(a)

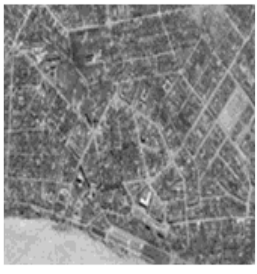

(d)

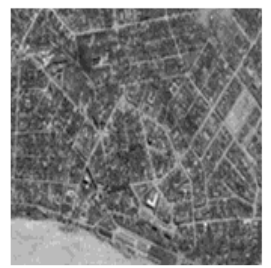

(b)

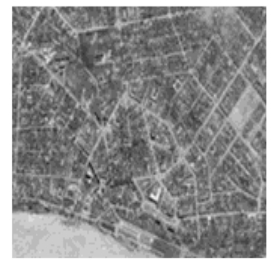

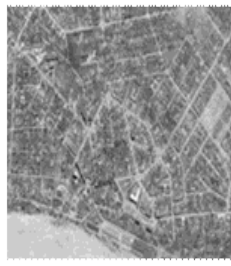

(c)
Figure 11 Satellite Images (a) original low resolution image, (b) DWT based decomposition and bi-cubic interpolated, (c) DWT based decomposition and GMM interpolated image, (d) NDWT based decomposition and bi-cubic interpolated Image and (e) NDWT based decomposition and GMM interpolated image.

\section{Conclusion}

A new Satellite image resolution improvement technique is achieved by using non-decimated wavelet transform to decompose the image and Gaussian Mixture Model interpolation to interpolate the coefficients. The results prove that the proposed technique is superior to existing methods. Image 1 is a Satellite Image of US-TOPO and Image 2 is a Satellite Image of Washington-DC was used for evaluating the methods. Table 1 lists the PSNR and Q Index achieved for the various techniques. To evaluate the proposed technique's performance, the original image was down sampled $50 \%$ on both the axes to obtain the down sampled image which is $1 / 4$ th size of the original image. After interpolation PSNR is computed using the original image as the base. 
Table 1: PSNR and QI results for proposed technique compared with conventional image resolution enhancement techniques.

\begin{tabular}{|l|c|c|c|c|c|c|c|c|}
\hline \multirow{2}{*}{ Technique } & \multicolumn{5}{|c|}{ PSNR (dB) } & \multicolumn{4}{c|}{ Q Index } \\
\cline { 2 - 9 } & Image 1 & Image 2 & Image 3 & Image 4 & Image 1 & Image 2 & Image 3 & Image 4 \\
\hline WZP & 25.97 & 25.68 & 24.76 & 24.73 & 0.9362 & 0.9218 & 0.9289 & 0.9167 \\
\hline Bilinear & 28.78 & 27.8 & 28.13 & 26.72 & 0.9483 & 0.9327 & 0.9434 & 0.9245 \\
\hline Bicubic & 27.60 & 26.15 & 27.18 & 24.75 & 0.9578 & 0.9405 & 0.9506 & 0.9348 \\
\hline $\begin{array}{l}\text { DWT } \\
\text { \&Bicubic }\end{array}$ & 28.64 & 27.26 & 27.52 & 26.61 & 0.9701 & 0.9525 & 0.9654 & 0.9451 \\
\hline $\begin{array}{l}\text { DWT \& } \\
\text { GMM }\end{array}$ & 30.44 & 29.48 & 29.49 & 28.01 & 0.9798 & 0.9641 & 0.9742 & 0.96 \\
\hline $\begin{array}{l}\text { NDWT \& } \\
\text { Bicubic }\end{array}$ & 29.78 & 29.02 & 28.08 & 27.47 & 0.9889 & 0.9716 & 0.9833 & 0.9653 \\
\hline $\begin{array}{l}\text { Proposed } \\
\text { Method } \\
\text { (NDWT } \\
\text { \&GMM) }\end{array}$ & 32.49 & 31.92 & 31.85 & 30.66 & 0.996 & 0.9838 & 0.9874 & 0.9776 \\
\hline
\end{tabular}

It is observed that the proposed NDWT and GMM method achieves higher PSNR and Q Index than the existing methods. The proposed method improves PSNR in the range of $6.52 \%$ to $22.31 \%$ compared to the existing techniques of image 1 . Similarly for other images the proposed methods performs in a better way.

\section{References}

[1] Hasan Demirel and Gholamreza Anbarjafari, "Satellite Image Resolution Enhancement Using Complex Wavelet Transform", IEEE Geoscience and Remote Sensing Letters, VOL. 7, NO. 1, January 2010.

[2] Muhammad Zafar Iqbal, Abdul Ghafoor Adil Masood Siddiqui, "Satellite Image Resolution Enhancement Using Dual - Tree Complex Wavelet Transform and Nonlocal Means", IEEEgeoscience and remote sensing letters, VOL. 10, NO. 3, May 2013.

[3] Hasan Demirel and Gholamreza Anbarjafari, "Discrete Wavelet Transform-Based Satellite Image Resolution Enhancement", IEEE Transactions on Geoscience and Remote Sensing, Vol 49, No. 6, June 2011.

[4] D. Hasan, F. Gholamreza, "Image Resolution Enhancement by Using Discrete and Stationary Wavelet Decomposition", IEEE Transactions on Image Processing, Volume: 20, NO. 5, 2011.

[5] T. V. Hymalakshmi, M. Praveen kumar "Image Super Resolution Model Using Fuzzy Rule", International Journal of Advanced and Innovative Research, Volume 4 Issue 7

[6] Ahire Rina B, V. S. Patil“'Overview of Satellite Image Resolution Enhancement Techniques" IEEE Conference Publications, Publication Year 2014., pages: Geoscience And Remote Sensing Letters, VOL. 7, NO. 1, january 2013.

[7] Battula. R. V. S. Narayana and K. Nirmala, "Image Resolution Enhancement by Using Stationary and Discrete Wavelet Decomposition", International
Journal of Modern Engineering Research (IJMER), Sep.-Oct. 2012, Vol. 2, Issue. 5, pp-3553-3555.

[8] E. Mohan, K. B. Jayarraman, U. Maheswaran, D. Sathiyaraj. G. Dhakshanamoorthi, "A Novel Approach for Satellite Image Resolution Enhancement “, International Journal of Engineering and Advanced Technology (IJEAT) ISSN: $2249-$ 8958, Volume-2, Issue-4, April 2013.

[9] ShirnSalehi and HomayounMahdaviNasab, "New Image Interpolation algorithm based on Dual Tree Complex Wavelet Transform and Multilayer Feed forward Networks", International Journal of Innovative Computing, Information and control, Volume 8, Number 10(A), October 2012, pp-68856902.

[10] André Jalobeanu, Laure Blanc-Féraud, and Josiane Zerubia, Fellow, "An Adaptive Gaussian Model for Satellite Image Deblurring", IEEE transactions on image processing, vol. 13, no. 4, April 2004, pages $613-621$.

[11] Lei Zhang, X. W. (AUGUST 2006). "An EdgeGuided Image Interpolation Algorithm via Directional Filtering and Data Fusion", IEEE TRANSACTIONS ON IMAGE PROCESSING, VOL. 15 , NO. 8.

[12] Huayong He, Jianhong Li, and XiaonanLuo, "Single image super-resolution using Gaussian Mixture Model", IEEE International Conference on Pattern Recognition, Nov 2012.

[13] MaximeTaquet, BenoitScherrer, Christopher Benjamin, Sanjay Prabhu, BenoitMacq, Simon K. Warfield, "Interpolating Multi-Fiber Models By Gaussian Mixture Simplification”, ISBI, 2012.

[14] Hou, X., Yang, J., Jiang, G., \& Qian, X. (2013). Complex SAR image compression based on directional lifting wavelet transform with high clustering capability. Geoscience and Remote Sensing, IEEE Transactions on, 51(1), 527-538.

[15] T. V. Hyma Lakshmi1, T. Madhu2, E. V. Krishna Rao3, V. Lakshmi Mounica4 "Satellite Image Resolution Enhancement Using Discrete Wavelet Transform And Gaussian Mixture Model", International Research Journal of Engineering and Technology (IRJET), Volume: 02 Issue: 04 | July2015

[16] Jean-Luc Starck, Jalal Fadili, and Fionn Murtagh, "The Undecimated Wavelet Decomposition and its Reconstruction ", IEEE TRANSACTIONS ON IMAGE PROCESSING, VOL. 16, NO. 2, FEBRUARY 2007.

[17] Khaled Ben Fatma, A. Enis Çetin:, "Design of Gaussian mixture models using matching pursuit",. NSIP1999: pages 466-470. 


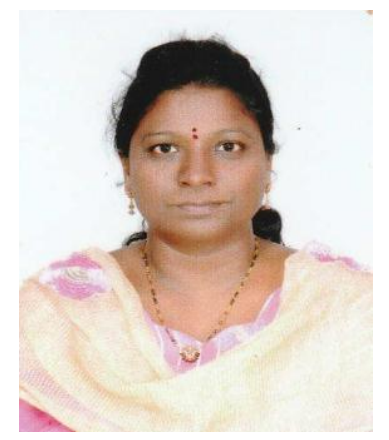

T. V. Hyma Lakshmi obtained her M. Tech. (ECE)., from JNTUCE, Ananthapur and B. E. (ECE) from S. R. K. R. Engg college, Bhimavaram. Presently working as Assistant Professor in S. R. K. R. Engg college, Bhimavaram and pursuing Ph. D. Program from K L University.

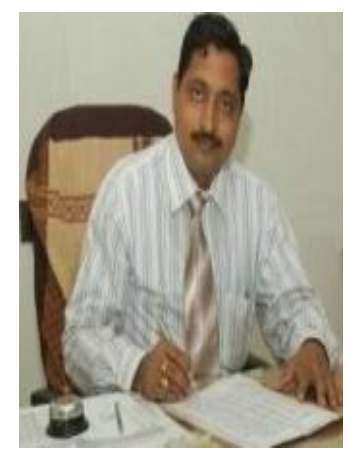

Dr. Tenneti Madhu obtained his B. E. degree from University of Madras, M. Tech from REC, Kurukshetra in 1994 and PhD from Osmania University in 2004. His research interests include GPS Data Analysis, Image Processing, Nano Technology and VLSI design. Presently working as Principal in S. I. E. T, Narsapur.

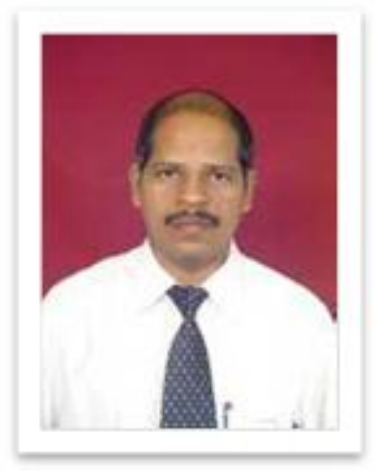

Dr. E. V Krishna Rao obtained his B. E. degree from Nagarjuna University, M. Tech from University of Delhi South Campus and PhD from JNTU. His research interests include Speech Processing, Signal Processing and Image Processing. Presently working as Professor in L B R College of Engineering, Mylavaram. 